\title{
Frontières
}

\section{Politique et religion chez les Toraja en Indonésie}

\section{Franck Michel}

Volume 19, numéro 1, automne 2006

Enjeux politiques et mort

URI : https://id.erudit.org/iderudit/016638ar

DOI : https://doi.org/10.7202/016638ar

Aller au sommaire du numéro

Éditeur(s)

Université du Québec à Montréal

ISSN

1180-3479 (imprimé)

1916-0976 (numérique)

Découvrir la revue

Citer cet article

Michel, F. (2006). Politique et religion chez les Toraja en Indonésie. Frontières, 19(1), 61-68. https://doi.org/10.7202/016638ar

\section{Résumé de l'article}

À Sulawesi, en Indonésie orientale, protégés par une région montagneuse et isolée, les Toraja Sa'dan ont subi la colonisation hollandaise plutôt tardivement (à partir de 1905). En dépit des conflits interreligieux qui ont marqué le XXe siècle, le syncrétisme constitue aujourd'hui encore une réalité quotidienne, rassemblant notamment les chrétiens (protestants, catholiques, pentecôtistes...), les musulmans et les derniers pratiquants des cultes autochtones. Le rapide essor du tourisme a ébranlé les valeurs et les habitudes des villageois, tout en proposant une autre voie d'affirmation de l'identité culturelle des Toraja. C'est dans ce contexte que les fêtes " traditionnelles ", en particulier celles liées au culte des morts, connaissent une nouvelle vie, non sans transformations irrémédiables. La mise en tourisme de la société n'occulte pas encore la ritualisation du spectacle de la mort. Cette contribution évoque tour à tour l'histoire de la difficile relation entre politique et religion en terre toraja et les liens étroits entre politique, tourisme et sacré, à travers la culture et les cérémonies religieuses locales.
Ce document est protégé par la loi sur le droit d'auteur. L'utilisation des services d’Érudit (y compris la reproduction) est assujettie à sa politique d'utilisation que vous pouvez consulter en ligne.

https://apropos.erudit.org/fr/usagers/politique-dutilisation/ 


\section{Résumé}

À Sulawesi, en Indonésie orientale, protégés par une région montagneuse et isolée, les Toraja Sa'dan ont subi la colonisation hollandaise plutôt tardivement (à partir de 1905). En dépit des conflits interreligieux qui ont marqué le $X X^{e}$ siècle, le syncrétisme constitue aujourd'hui encore une réalité quotidienne, rassemblant notamment les chrétiens (protestants, catholiques, pentecôtistes...), les musulmans et les derniers pratiquants des cultes autochtones. Le rapide essor du tourisme a ébranlé les valeurs et les habitudes des villageois, tout en proposant une autre voie d'affirmation de l'identité culturelle des Toraja. C'est dans ce contexte que les fêtes "traditionnelles", en particulier celles liées au culte des morts, connaissent une nouvelle vie, non sans transformations irrémédiables. La mise en tourisme de la société n'occulte pas encore la ritualisation du spectacle de la mort. Cette contribution évoque tour à tour l'histoire de la difficile relation entre politique et religion en terre toraja et les liens étroits entre politique, tourisme et sacré, à travers la culture et les cérémonies religieuses locales.

Mots clés: Religion - fêtes - tourisme politique - mort - Indonésie syncrétisme.

\section{Abstract}

Sulawesi, in Asian Indonesia, is protected by a region of mountains, isolating the Toraja Sa'dan who were colonized quite late by the Dutch (starting in 1905). Despite inter-religious conflicts that marked the $20^{\text {th }}$ century, syncretism remains a daily reality, even today, bringing together Christians (Protestants, Catholics, Pentecostals, etc.), Muslims and practitioners of surviving native cults. The rapid increase of tourism has destabilized the values and customs of the villagers, while at the same time proposing another means to affirm Toraja cultural identity. It is in this context that the "traditional" festivals, especially those relating to the cult of the dead, have regained in popularity, not without some important transformations. Rendering their society a tourist attraction has not yet destroyed the ritualization of the spectacle of death. This paper evokes the difficult history of relations between politics and religion on Toraja soil, and the close ties between politics, tourism and the sacred developed through culture and local religious ceremonies.

Keywords: Religion - festival tourism - politics - death Indonesia-syncretism.

\section{POLITIQUE ET RELIGION CHEZ LES TORAJA EN INDONÉSIE}

Franck Michel,

anthropologue et enseignant, Université de Corse.

En Indonésie orientale, les Toraja Sa'dan résident essentiellement dans le département (kabupaten) qui porte leur nom (Tana Toraja, également contracté en Tator, ce qui signifie «Terre des Toraja») et qui se situe dans la province de Sulawesi-Sud. La colonisation hollandaise s'est imposée tardivement (1905-1907) dans cette région montagneuse, isolée et difficile d'accès. Mais l'évangélisation connut un «succès », certes lent, mais durable, en partie à cause de la présence de puissants voisins musulmans. Si les conflits interconfessionnels furent nombreux, le syncrétisme religieux constitue aujourd'hui une réalité quotidienne, rassemblant notamment les chrétiens (protestants, catholiques, pentecôtistes...), les musulmans et les derniers pratiquants des cultes autochtones. Le rapide essor du tourisme a ébranlé les valeurs et les habitudes des villageois. Il a aussi permis aux habitants de gérer alternativement leur futur, leurs politique et économie locales, ainsi que d'affirmer leur identité culturelle. Les croyances religieuses autochtones exigent un investissement considérable et relèvent d'un mode de vie et de pensée en lien étroit avec la "voie des ancêtres». La majorité des Toraja sont aujourd'hui chrétiens mais, en dépit des conversions et des changements socioculturels irrémédiables - notamment ceux attribuables à l'ingérence de l'État indonésien dans les affaires locales, à la mondialisation et au tourisme international- qu'ils connaissent depuis quelques décennies, les faits culturels et religieux continuent d'occuper une place essentielle comme le montrent, par exemple, les cérémonies funéraires «traditionnelles ». Le monde des Toraja se divise en deux sphères distinctes ainsi que les ont fixées conjointement la coutume (ada'ou adat) et la religion (aluk ou agama) : l'Est et l'Ouest, le matin et le soir, les rites funéraires (Rambu Solo') et les rites propitiatoires $\left(\right.$ Rambu Tuka') ${ }^{1} \ldots$

Les fêtes toraja attirent et fascinent les touristes autant que les autochtones. La mise en tourisme de la société n'occulte pas encore la ritualisation du spectacle de la mort. Même si certains témoignages recueillis auprès de touristes présents à une fête Merok (Siguntu', 1996) sont sans appel: «ça ressemble à une kermesse», dit l'un 
de ces voyageurs démysthifiés; «il n'y a plus grand-chose d'authentique dans toute cette mise en scène touristique » ajoute un autre... Les invocations et les critiques de ce genre reviennent parfois dans la bouche des visiteurs de passage assistant à une fête toraja, passage certes hâtif, mais qui n'en reste pas moins, pour la plupart d'entre eux, marqué par le sceau de l'authenticité et perçu comme le «clou» de leur séjour en Indonésie. Cet article reviendra d'abord sur l'histoire de la difficile relation entre politique et religion en terre toraja, puis évoquera les liens étroits entre politique, tourisme et sacré à travers la culture et les cérémonies religieuses locales.

\section{HISTOIRE, POLITIQUE ET RELIGION}

Avant d'aborder l'histoire politique et religieuse, quelques précisions à propos de l'Aluk todolo, culte autochtone des morts, et de son interaction avec le dogme chrétien imposé de l'extérieur nous montrent d'emblée comment les Toraja, traditionnellement opposés à leurs voisins Bugis musulmans, se contraindront bon gré mal gré à une forme de syncrétisme exigée par la situation coloniale hollandaise, puis régionale indonésienne. Le christianisme s'est durablement installé chez les Toraja dès 1913, avec l'arrivée de la première mission protestante à Rantepao. Il connaît cependant peu de succès à ses débuts, et à partir de 1937, s'ajoute un autre obstacle à une conversion rapide des autochtones: les rivalités entre les missions protestantes et catholiques. Les catholiques, se manifestant surtout après la Seconde Guerre mondiale, entendent partager le «pouvoir spirituel» avec les protestants hollandais (et désormais aussi toraja), déjà bien installés. Durant la courte phase de la présence japonaise (mars 1942 à automne 1945), il est pratiquement mis fin aux différentes activités missionnaires (écoles, catéchisme, etc.). Il faut ici relever que la cruauté des troupes japonaises a plutôt contribué à rapprocher les Toraja des chrétiens hollandais. De la même manière, le nouveau gouvernement indonésien n'a pas su immédiatement comprendre la spécificité de la situation locale. Pendant une vingtaine d'années, l'attitude quelque peu simpliste et centralisatrice adoptée par l'État indonésien consiste ainsi à ne reconnaître que trois religions « officielles» : islam, christianisme, religion «balinaisehindouiste ». C'est seulement en 1969, après de longues et houleuses discussions, que le Parlement national reconnaît officiellement la religion toraja (parmi d'autres aussi). L'Aluk todolo sera dorénavant considéré comme une religion à part entière, même s'il se retrouve inscrit dans la Constitution au sein du groupe dit des «religions bali-

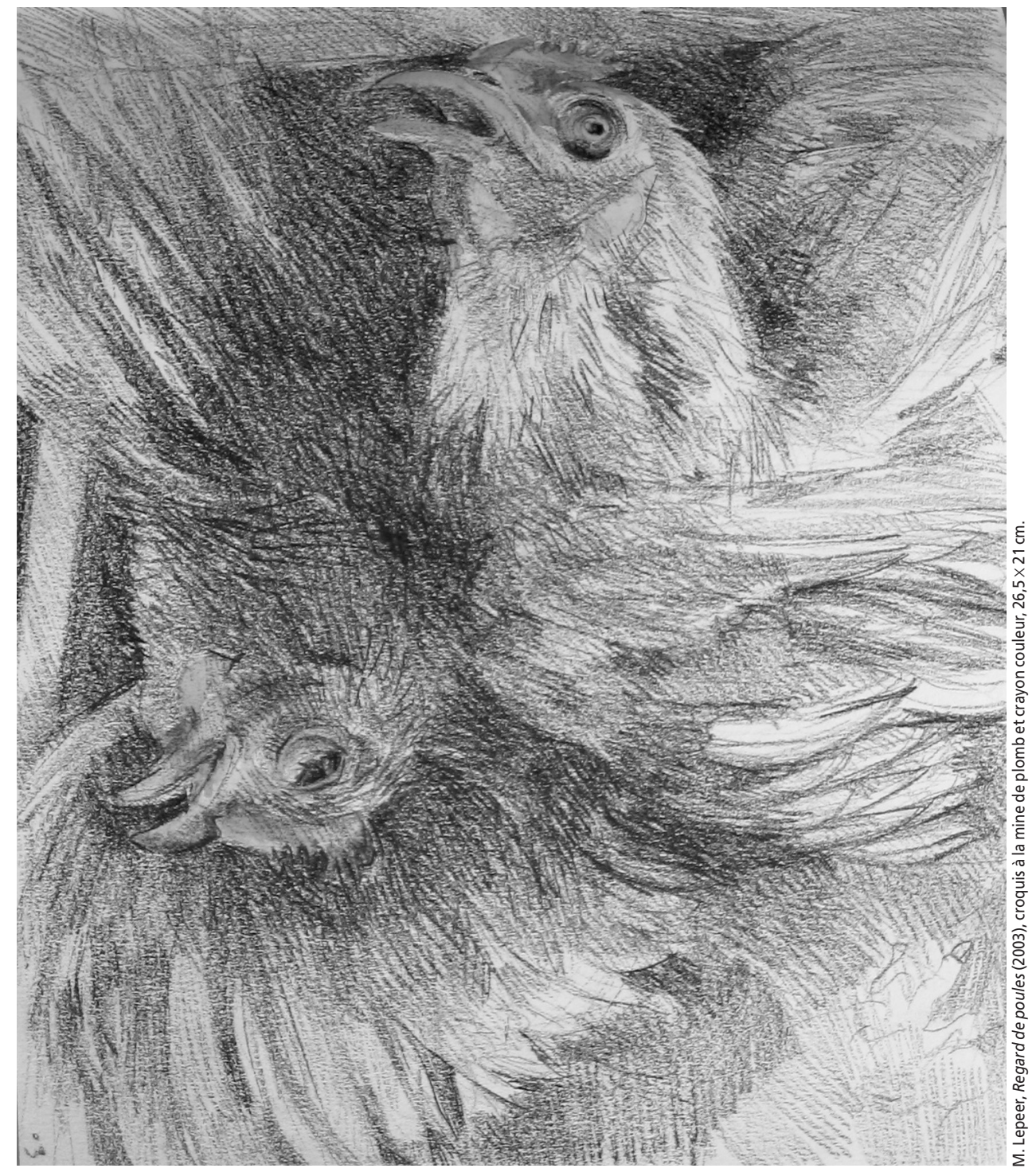

naises et hindouistes » (Agama Bali ou Hindu Dharma), ce qu'il n'est manifestement pas! Évoquons à présent brièvement l'histoire politique et religieuse des Toraja entre 1950 et nos jours.

Après des années de guerres internes, puis de colonisation hollandaise et d'évangélisation forcée, le pays toraja a dû subir les affres de l'occupation japonaise et s'est retrouvé ensuite dans une région déchirée par la lutte pour l'indépendance ${ }^{2}$. Au cours des années 1950, le nouveau gouvernement indonésien entreprend des réformes importantes, notamment dans les domaines de l'éducation et de l'administration, mais il faut admettre qu'en raison de l'immensité de l'archipel et de la difficulté d'accès, le pays toraja ne bénéficie que très lentement de certaines de ces mesures. D'autre part, durant plus d'une décennie, le climat politique local est plutôt anarchique; on peut relever en premier lieu l'exaspération des révoltes qui perdurent entre les rebelles musulmans et les nouveaux représentants toraja, en général chrétiens et intégrés au gouvernement de la jeune nation.
La principale rébellion, dirigée par un musulman de Luwu', dont le nom est Kahar Mudzakkar, a un impact extrêmement fort, et ne prendra fin que vers le milieu des années 1960. Le mouvement panislamique Darul Islam Tentara Indonesia, par le biais des troupes de Kahar Mudzakkar, tente d'interdire brutalement la pratique de la religion traditionnelle des Toraja (et la consommation de porc) et d'imposer par la force ses conceptions religieuses. Reprenant un article du New York Times, daté du 11 mars 1951, Tibor Mende évoque ces événements dans les termes suivants: «Les rebelles sont pour la plupart des hommes qui se battaient autrefois contre les Hollandais. » Il présente Kahar Mudzakkar comme un "célèbre chef de guérilla antihollandaise» et précise que les rebelles «sont mécontents de voir des hommes ayant servi naguère contre les Républicains dans les forces coloniales hollandaises faire désormais partie de l'armée indonésienne et mécontents aussi de la présence dans l'administration d'anciens fonctionnaires 
des Hollandais ${ }^{3} »$. Avec le recul, cette reconstitution paraît aléatoire sur un plan historique, mais elle nous éclaire utilement sur l'atmosphère très tendue à cette époque dans les montagnes toraja.

Le lieutenant de Kahar Muzdakkar qui joue un rôle prééminent à Tana Toraja est Andi Sose, natif de Duri mais étroitement lié aux familles princières de Tallu Lembangna (confédération au sud de Tator). À cette période, le Puang («seigneur» ou chef local) Andi Lolo de Makale retrouve son poste de leader du Tongkonan Ada', rebaptisé par le nouveau régime, Dewan Pemerintahan Sendiri (Conseil gouvernemental autonome) ; la nouvelle désignation du PARKI, principal parti confessionnel, devient PARKINDO en 1950, et le parti sera présidé par F. K. Sarungallo. Cette même année voit la formation d'un gouvernement d'urgence (Pemerintah Darurat). Localement, le Comité national indonésien (KNI) porte à sa tête un instituteur toraja, Rongne 4 , ancien "esclave» du Puang de Sangalla', résidant à Enrekang. F. K. Sarungallo, membre de l'aristocratie de Kesu, prend également activement part au gouvernement du KNI.

La conséquence majeure de cette période de troubles constants a été d'engager plus intensivement les Toraja sur la voie de la christianisation, de même qu'elle a remis à l'ordre du jour les vieilles querelles de voisinage entre les Bugis «islamisés et expansionnistes» et les Toraja. Cette animosité entre les deux communautés, stimulée sinon entretenue par le climat de guerre civile, conduit à toujours plus de méfiance à l'égard de l'Autre et donne aux deux peuples des alibis pour se réfugier derrière leurs propres certitudes. De 1950 à 1965, la situation matérielle des habitants de Tana Toraja reste particulièrement précaire, la population ne cesse d'augmenter alors que les terres cultivables viennent à manquer de manière inquiétante, dans une région où l'autosuffisance alimentaire constitue de longue date un souci permanent.

Des conflits de «classes » se greffent sur ceux de «castes » à propos, surtout, du partage des terres et des relations entre grands propriétaires, petits exploitants et « ouvriers agricoles » : en somme, un problème crucial que connaissent de nombreuses régions du tiers monde alors récemment émancipées. C'est à ce moment que des sections du parti communiste indonésien (PKI) parviennent à recruter des adeptes au sein de la population paysanne souvent déshéritée de Tana Toraja. Dans le contexte religieux, lors de la pratique de certains rituels (par exemple le $\left.M a^{\prime} b u a^{\prime}\right)$, certains chefs toraja prônent davantage d'égalité, notamment pour le partage de la viande (Volkman, 1985, p. 41-43). Aussi, le monde des Toraja va se trouver soudainement bouleversé par son intégration au sein de l'espace géographique et surtout politique indonésien, ainsi que le montrent les manifestations et l'esprit de révolte qui s'étendent dans la région. En quelque sorte, l'arrivée du PKI survient à point nommé, au moment où celui-ci profite du soutien qu'il apporte aux luttes paysannes; les dirigeants du PKI, Selenda, et même le Secrétaire général du Parti, Aïdit, visitent le pays toraja au début des années 1950, et y gagnent une certaine popularité (la structure sociale toraja, «inégalitaire» par excellence, représente il est vrai un terreau de choix!), acquise d'ailleurs assez curieusement davantage dans les rangs de l'élite éduquée qu'auprès des «masses paysannes ${ }^{5}$ ».

Dès 1953, notamment après «l'incident du 4 avril », les divers mouvements de guérilla sont très divisés et perdent lentement tout crédit aux yeux de la population toraja, qui apporte sa confiance de plus en plus au PARKINDO. En effet, ledit incident du 4 avril 1953, engageant une confrontation serrée entre, d'un côté, Andi Sose et Frans Karagan et leurs partisans, et, de l'autre, les troupes du contingent de Palopo-Masamba soutenues par une majorité de Toraja, est finalement un succès pour les Toraja chrétiens. Une victoire qui signale autant l'installation progressive mais incontestable des forces politiques chrétiennes que l'affermissement de l'identité toraja.

Cela étant, les disputes locales perdurent et s'amplifient même en ce qui concerne les problèmes des terres et surtout des rizières. À Sulawesi-Sud, les guérillas connaissent alors une islamisation de plus en plus grande de leurs effectifs (sur le plan national aussi, on note la création de l'Armée indonésienne de l'Islam, corps militaire regroupant de nombreux mouvements de guérilla). Agressions, exactions en tout genre, incendies de villages, etc., se multiplient en terre toraja, et les pratiquants de l'Aluk todolo sont à cette période les victimes les plus importantes (les deux seules religions «autorisées » par les assaillants se résumant à l'islam et au christianisme).

Inéluctablement, l'essor du mouvement Darul Islam engendre une série de conséquences tragiques chez les Toraja. C'est dans ce climat d'insécurité que se préparent les premières élections générales en 1955. De nombreux partis nouvellement créés sont en compétition: "Alors que beaucoup de Toraja sont impliqués dans les groupes fonctionnels du PKI, leur allégeance politique allait finalement au PARKINDO. Chez les Toraja, christianisme et socialisme ne s'excluaient pas mutuellement, ce qui à la fois aidait et donnait la possibilité au PKI de s'organiser. » Les deux partis religieux les plus «fondamentalistes » sont le MASYUMI (musulman) et le PARKINDO (chrétien protestant). Ce dernier remporte un vif succès à ces élections, obtenant cinq des sept sièges à pourvoir au Parlement régional de Luwu', et recueillant plus de $50 \%$ des votes 6

Le PARKINDO va diriger durant une quinzaine d'années la vie politique à Tana Toraja. Trois raisons majeures expliquent ce résultat électoral assez exceptionnel du PARKINDO : c'est d'abord un parti religieux qui représente les chrétiens majoritaires dans la région; c'est ensuite un parti politique apportant surtout une réponse régionale au pouvoir croissant du «centre» javanais; c'est enfin un parti «identitaire» qui veut incarner la préservation de la société, de la culture et des traditions autochtones, en un mot de l'identité toraja. Ces quelques aspects expliquent la popularité et les succès durables enregistrés localement par le PARKINDO et par ses deux «annexes» que sont l'Église protestante toraja (Gereja Toraja) et la Fondation pour l'enseignement chrétien toraja (YPKT). Avant le coup d'État militaire amenant le clan Suharto au pouvoir à Jakarta, le parti chrétien protestant aura l'occasion de tester localement ses forces lors de la rébellion de 1958 connue sous l'appellation de PERMESTA; celle-ci est fomentée par des militaires «régionalistes» et se développe à partir de Makassar. Le PARKINDO négocie mystérieusement avec les «frères » minahasa du nord de l'île (qui sont proches des rebelles), mais ne s'engage pas en profondeur dans cette aventure.

Une terrible et sanglante chasse aux communistes est déclenchée sur tout le territoire indonésien dès 1965 : quelques Toraja seront tués à cette occasion et beaucoup d'autres arrêtés, à tel point qu'aujourd'hui encore - soit sept ans après la chute du dictateur Suharto - le terme de «communiste» est comme absent du vocabulaire, et le réflexe de la peur toujours présent... L'Ordre Nouveau du président Suharto s'était, depuis la fin des années 1960, nettement imposé et le pays toraja, à l'instar de l'ensemble de l'Indonésie, s'est profondément ouvert sur l'extérieur. Les investissements étrangers, l'industrie touristique, le développement économique érigé sur le modèle occidental, les divers objectifs et résultats issus de l'effort intensif de modernisation nationale, touchent et concernent irrémédiablement les Toraja. Sur le plan politique et diplomatique, rien de mieux qu'un régime stable, fut-ce une dictature aux mains sales, mais bien trempées dans le marché mondial. Pour le régime, favoriser l'essor du religieux constitue une voie pour éviter la révolte politique. Cela reste valable en 2006, en dépit du danger que représente aujourd'hui la surenchère religieuse, voire l'ethnicisation des conflits, orchestrée par le pouvoir. 
Retour à la fin des années 1960: pour le peuple toraja, une situation nouvelle est à découvrir et à négocier avec intelligence par les protagonistes, celle d'exister pleinement dans un monde encore régi par des valeurs souvent contraires aux leurs. À ce sujet, dès 1966, Sutan Takdir Alisjahbana écrit ces propos encore d'actualité (même si opposer aujourd'hui tradition et modernité n'a plus guère de sens) : «Dans certains endroits, de nouvelles valeurs et normes ont émergé, remplaçant les anciennes, et apportant des orientations et un dynamisme renouvelé pour la société indonésienne. [...] Toutefois, il y a aussi beaucoup d'individus et de groupes qui ont perdu tout sens de direction dans le fossé entre les deux grands courants culturels [tradition et modernité]. Pour ces gens, l'ancien est mort et le neuf n'est pas encore parvenu à s'installer»(Alisjahbana, 1966, p. VIII). Depuis 1969 et surtout 1972, Tana Toraja vit au rythme d'une modernisation allant sans cesse en s'accroissant. Parallèlement, le tourisme commence timidement à s'installer à partir de ces années. Après la modernisation politique et économique, dont le tourisme international est dorénavant devenu la composante majeure, les Toraja ont encore à affronter d'autres facteurs de changements essentiels, notamment dans la vie sociale et religieuse.

Si dans le champ politique, l'ère Sukarno est chez les Toraja plutôt l'ère du PARKINDO, cette tendance se traduit également dans le domaine religieux. Au cours des troubles des années 1952-1953, de nombreux Toraja, principalement de religion Aluk, se convertissent plus ou moins de force à l'islam, mais c'est le christianisme qui va, de très loin, attirer le plus grand nombre de Toraja au cours de cette même période (surtout à l'ouest du pays). Forte du poids et du soutien apportés par le PARKINDO et le YPKT, qui créent de nombreuses écoles à partir des années 1950, l'Église protestante toraja (Gereja Toraja) ne cesse d'augmenter son pouvoir et son prestige, multipliant soudainement le nombre de ses adeptes.

Ces conversions sont rapides et massives, elles ne masquent pas moins également des intentions conjoncturelles; en l'occurrence, éviter les exactions des bandes de rebelles, éviter qu'elles saccagent les villages et tuent les porcs, etc. Il fallait en outre "adhérer» à l'une des deux seules religions officiellement reconnues: l'islam ou le christianisme. Du fait du travail des colonisateurs et bien sûr des missionnaires hollandais, et surtout du lourd héritage de leur passé incluant la haine séculaire de leurs voisins musulmans (même si elle n'est pas historiquement et culturellement fondée), les Toraja vont largement opter pour la religion chrétienne. Ainsi, entre 1950 et 1965 , les conversions progressent de $10 \%$ par an pour la seule Gereja Toraja; on passe ainsi en quinze ans de 23000 baptisés à environ 90000 (Bigalke, 1981, p. 434)! Dans sa thèse, Eric Crystal évoque précisément la forte vague de christianisation, et les motivations particulières qui l'ont entraînée, en seulement une quinzaine d'années après la Seconde Guerre mondiale, plus exactement entre la fin des années 1940 et le début des années 1960 (Crystal, 1970).

En dépit d'une certaine progression enregistrée dès les années 1950, l'Église catholique et les «petites» sectes protestantes devront encore patienter jusqu'à l'aube des années 1970, avant de voir leur rôle et leur pouvoir régional s'affirmer auprès des habitants toraja. Les sectes protestantes, au premier chef desquelles figurent les mouvements pentecôtistes, très opposées à tout ce qui ressemble à de l'Aluk todolo, acquièrent, auprès des Toraja, depuis les années 1980 et surtout 1990, une plus grande audience que d'aucuns jugent inquiétante. Évidemment, toutes ces conversions, même si en partie elles sont à inscrire dans la catégorie «chrétien de peau» (sarani balulang), contribuent à modifier de manière considérable le paysage religieux toraja, et avec lui les habitudes et le mode de vie des Toraja ayant embrassé l'Aluk Belanda, autrement dit la foi de l'ancien colonisateur (Belanda $=$ Hollandais).

D'une certaine manière, il n'est pas faux de dire que depuis les années 1970, les chrétiens occupent quasiment tous les postes de responsabilité et de décision à Tator, et la société en mutation l'est d'abord de leur fait. Les secteurs politique (avec le PARKINDO), éducatif (avec l'association YPKT) et cultuel (avec Gereja Toraja) sont presque exclusivement aux mains des chrétiens et, plus exactement, des protestants. Ce sont d'abord eux qui gèrent les changements en cours durant ces dernières décennies (dont les quatre pôles majeurs peuvent être représentés par la télévision, l'Église, le tourisme et l'école), avec l'assentiment et le concours du gouvernement indonésien. Dans les années 1990, puis 2000, les Toraja soutiennent majoritairement le PDI (puis le PDI-P), parti certes nationaliste, mais accueillant favorablement en son sein les minorités chrétiennes, dont la patronne a longtemps été Megawati Sukarnoputri, future présidente de l'Indonésie et fille de Sukarno, "père de l'indépendance». C'est dans la continuité que les Toraja ont associé identité politique avec identité religieuse, sans jamais perdre de vue leur identité culturelle... Mais ne sont-ce pas aujourd'hui les chrétiens qui se disent et se font les plus ardents défenseurs de l'identité toraja... qu'ils ont pourtant eux-mêmes mise à mal ? Une contradiction pas toujours facile à assumer.

\section{STRATÉGIES POLITIQUES ET TOURISME RELIGIEUX}

Depuis une vingtaine d'années déjà, le tourisme apparaît comme une valeur économique sûre et rentable aux yeux des Toraja, en particulier auprès des dirigeants politiques et des acteurs économiques locaux. Les responsables religieux entendent aussi bénéficier de cette manne quitte à fermer les yeux sur certaines pratiques animistes trop utiles au maintien de la tradition... Les chiffres attestent de l'essor rapide et continu des arrivées de touristes dans le département de Tana Toraja, malgré un certain tassement ces dernières années (crise politique et attentats en Indonésie...). On notera au passage que les touristes étrangers les plus nombreux à se rendre chaque année au pays toraja sont les Français, avec environ 25000 voyageurs par année depuis 1995. Depuis la chute de Suharto en mai 1998, l'instabilité politique qui s'en suivit et les récentes vaques d'attentats, les touristes se font plus discrets, mais semblent aujourd'hui revenir (devant la force des désirs d'ailleurs, on s'habitue à tout, même au terrorisme!)...

Il y a plus de vingt ans, c'est-à-dire au début de la déferlante touristique, un article anonyme paru dans Le Monde avait été consacré aux fêtes toraja et à l'essor du tourisme dans cette région? ${ }^{7}$. L'auteur y dénonçait déjà la recherche de l'authenticité à tout prix: "Pas de limite au sensationnel. Les marchands de voyage sont en train de découvrir un nouveau filon: les sacrifices religieux. » La quête d'exotisme et de sensations fortes attire des touristes occidentaux blasés des circuits standardisés. Mais, finalement, l'attrait des adeptes de tous les types de tourisme confondus ne se résumet-il pas à une quête de spiritualité ? Venant pour la majorité d'Occident, où la laïcité mêlée aujourd'hui à la mutation économique et au défi de la mondialisation angoisse quantité d'âmes errantes et orphelines, les touristes étrangers recherchent très souvent à réinsuffler leur philosophie de la vie ainsi que leur religiosité déliquescente. Surtout, les fêtes occidentales, banalisées et tournées vers la consommation, n'ont plus guère de points communs avec ces fêtes sacrées toraja, forcément sacralisées par les regards assoiffés en provenance de l'étranger... Pèlerin des temps modernes, le touriste fuit temporairement son univers connu et banal pour mieux assurer sa propre survie. Ainsi que celle de son monde si méprisé. Attitude paradoxale mais historiquement maintes fois prouvée...

Tana Toraja, nouvelle destination du «tourisme religieux»? On s'interroge évidemment sur ce qui ressemblerait à un tourisme dit «religieux». N'est-ce pas au moment où la pratique religieuse décline - 


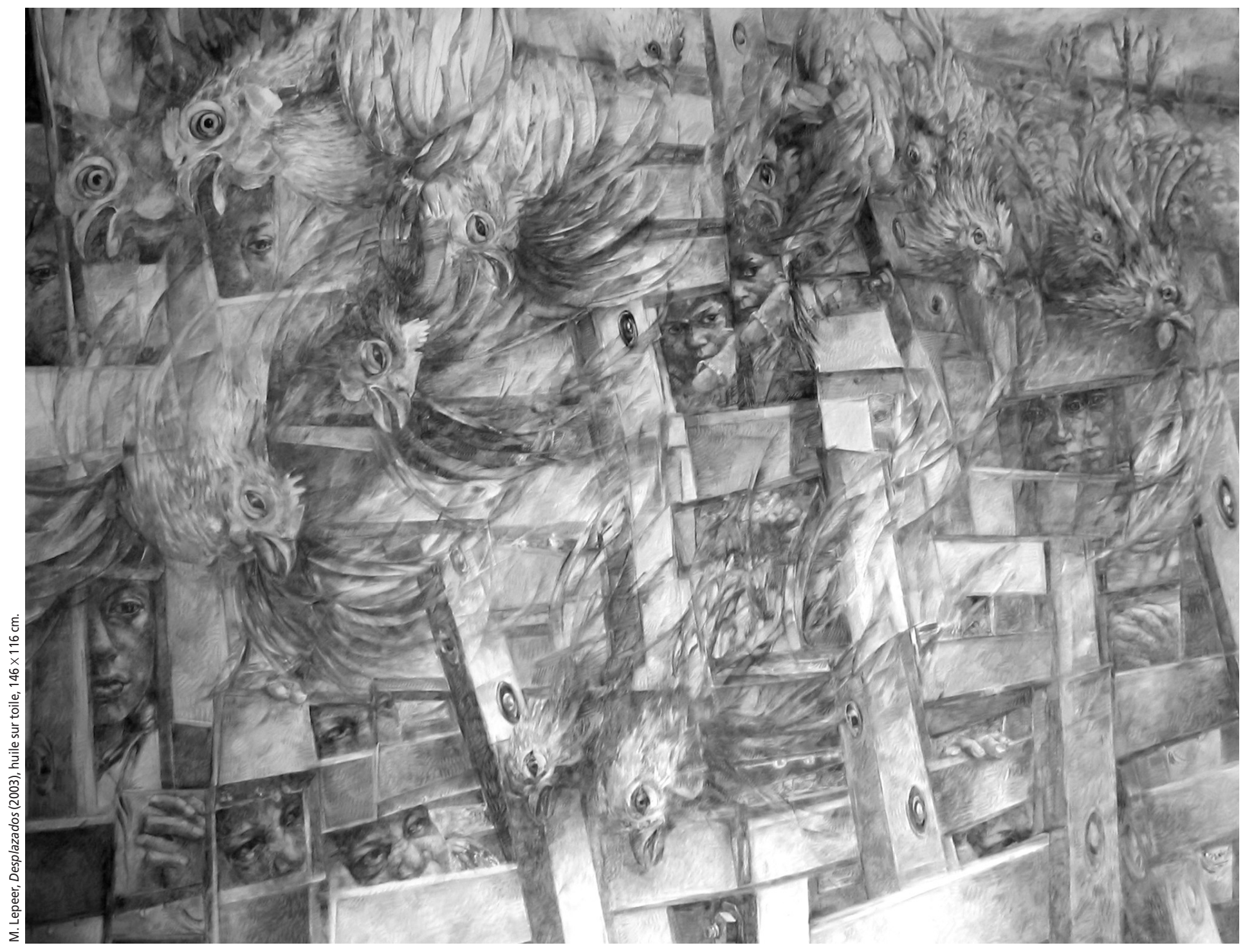

ou parfois se radicalise- dans un Occident non seulement en quête de références mais aussi de spiritualité, que les visiteurs recherchent chez les Toraja la pratique du sacré dans la ferveur des solennelles cérémonies funéraires? En réalité, les Occidentaux observent, avec un sentiment de nostalgie à peine dissimulé, les autochtones pratiquer ce qu'ils ne pratiquent plus. Le pèlerin laïc et moderne est surtout un adepte fervent $\mathrm{du}$ tourisme sacré et traditionnel: œuvre collective, le voyage est toujours un pèlerinage. La fête recherchée par le touriste en est sans doute le meilleur exemple: effervescence pour Durkheim, système de reproduction des croyances et des mythologies pour Frazer, institution coutumière pour les autochtones, attraction vivante et curiosité folklorique pour les visiteurs, la fête est d'abord un acte collectif ${ }^{8}$. À l'image du tourisme qui s'articule essentiellement dans un univers occidental, la fête s'inscrit chez les Toraja, comme en d'autres lieux, dans un espace-temps non ordinaire matérialisant la rupture avec l'habitude, la routine, le quotidien. À vocation communautaire et rituelle pour les Toraja, spectaculaire et exotique pour les touristes, la fête remplit bien sa fonction primordiale de rupture momentanée de l'ordre social établi et convient par conséquent à toutes les parties concernées.

Nous dirons qu'un pèlerinage n'est totalement réussi et complet que dans la mesure où il est accompagné de cérémonies. En tant que rupture collective avec la vie et le travail quotidiens, les fêtes marquent des temps forts dans la vie des Toraja, elles sont nombreuses et fréquentes, même si les fêtes funéraires constituent aujourd'hui de facto les cérémonies les plus importantes. Elles ne marquent pas seulement le temps mais aussi le sentiment d'appartenance collective; ainsi une fête peut "arrêter» ou retarder toute autre activité normale (par exemple, le passage sur une grande route des bus et des voitures est impossible lors d'une cérémonie funéraire dont les lantang ou « huttes temporaires » encombrent toute la route). En raison de leur importance aux yeux de la population locale, l'organisation des fêtes requiert des moyens considérables et des coûts prohibitifs. Ainsi un «chantier » pour un prestigieux Rambu Solo'peut monopoliser un millier d'ouvriers, occasionner la dépense d'une véritable fortune accumulée durant un demi-siècle, et ensuite accueillir plusieurs milliers d'invités dont souvent des personnalités importantes (du gouvernement de Jakarta par exemple). Pratiques religieuses et rituels politiques sont ici particulièrement imbriqués. La récupération politique des coutumes religieuses à des fins touristiques s'avère de plus en plus évidente.

À Tana Toraja, une cérémonie réussie est en premier lieu un événement extra-ordinaire dont on se souviendra et dont on parlera encore longtemps après l'événement. Et les sacrifices de porcs et plus encore de buffles occupent une place privilégiée dans le séjour au pays toraja pour la majorité des touristes. Pour les Toraja également mais pour d'autres raisons: le sacrifice consiste d'abord, comme l'ont bien relevé Hubert 
et Mauss il y a un siècle, à faire communiquer les dieux et les hommes (en facilitant notamment à l'âme du défunt l'accès à Puya ou «paradis toraja»), mais il permet aussi de remettre chaque membre de la communauté à sa place. Cette étape est franchie grâce à l'acquittement de dettes envers un ou plusieurs ancêtres ${ }^{9}$. Pour les touristes, l'acte sacrificiel - caché ou honteux (combien de tour-opérateurs guident les pas de leurs clients vers nos abattoirs?) - étonne et libère les instincts, comme l'attestent avec force les témoignages recueillis auprès des touristes. Leur comportement n'est pas sans ambiguiité : certains racontent ainsi qu'ils ne «veulent pas et ne peuvent pas regarder ce massacre, cette boucherie ». Ils se déplacent pourtant à la fête au moment précis où commencent les sacrifices, et aucun de ceux qui se plaignent de «la barbarie des Toraja » n'oublie de photographier la scène si cruelle de ce macabre spectacle qui n'a, en principe, rien de touristique (ou alors les abattoirs restent une alternative touristique occidentale possible)...

Il faut bien évidemment mentionner l'introduction de rapports marchands nouveaux et surtout la pénétration du cycle de la monétarisation que l'industrie du tourisme n'est pas la seule à avoir fortement encouragée. Dans l'ensemble, le phénomène «inflationniste» que l'on observe dans les cérémonies n'est pas directement imputable à la présence des touristes. Il résulte essentiellement du plus grand flux monétaire et de richesses en tout genre qui circulent désormais en pays toraja. Un rang social élevé est en fait à la fois de plus en plus difficile à tenir (en raison de la concurrence et de la forte compétition, il faut toujours donner et partager davantage, ce qui conduit à une coûteuse surenchère pour de nombreuses familles) et de plus en plus facile à gagner (pour ceux qui ont fait rapidement fortune à l'extérieur et que l'on nomme ici les «nouveaux riches»). Les principales victimes de cette situation sont évidemment les anciens nobles, dont le prestige dépendait jusqu'alors du rang familial et non de l'argent. Dorénavant, la fortune est le meilleur marchepied pour s'élever socialement et donc politiquement au pays des ancêtres au balcon qui ne surveillent plus que de loin les sacrilèges mercantiles des vivants...

Les fêtes, dont l'organisation et le déroulement sont parfois affectés par ces nouvelles exigences, subissent également d'importantes transformations: choix des dates (souvent pendant les vacances de juillet) et des invités (surtout les personnalités officielles), danses plus spectaculaires (certains Ma'badong ou rondes funéraires), «activités spéciales» pour touristes, etc. Le «tourisme mortuaire à Tana Toraja»,

À VOCATION COMMUNAUTAIRE ET RITUELLE POUR LES TORAJA, SPECTACULAIRE ET EXOTIQUE POUR LES TOURISTES, LA FÊTE REMPLIT BIEN SA FONCTION PRIMORDIALE DE RUPTURE MOMENTANÉE DE L'ORDRE SOCIAL ÉTABLI ET CONVIENT PAR CONSÉQUENT À TOUTES LES PARTIES CONCERNÉES.

d'après le titre d'un article déjà ancien de T.A. Volkman ${ }^{10}$, est actuellement toujours en plein essor, et les conséquences de ce type de tourisme dit culturel peuvent, à terme, se révéler dramatiques. L'ingérence du politique et du marché au cœur des traditions est notamment prégnante. Des dysfonctionnements apparaissent également: ainsi, les Rambu Solo'deviennent les cérémonies souvent les plus importantes, alors que les Rambu Tuka'(à l'exception des Mangrara Banua) tendent lentement à disparaître (Merok, Maro', Ma'bua, Ma'bugi, etc.). Moins spectaculaires et plus compliquées... Moins efficaces donc!

Paradoxalement, ce sont quelquefois les Églises chrétiennes qui se chargent de perpétuer et de veiller à la préservation des traditions rituelles anciennes et préchrétiennes... Ainsi, en 1978, l'Église la plus puissante (Gereja Toraja) a officiellement protesté contre les excès perpétrés par les touristes et surtout contre l'aspect spectaculaire des rites. Si les rites traditionnels ne sont à l'heure actuelle jamais pratiqués à la seule intention des touristes, nous pouvons toutefois remarquer de temps en temps un côté «rite-spectacle» tout à fait superficiel et superflu ${ }^{11}$. En général, plus les invités sont nombreux, plus la cérémonie est considérée comme réussie (et le souvenir du défunt célébré), c'est pourquoi les touristes sont toujours, dans un premier temps du moins, les bienvenus. Les Toraja sont contents quand il y a beaucoup de fêtes: compte tenu de l'argent que cela rapporte, des échanges économiques que cette situation développe (taxes pour le gouvernement, règlement des dettes familiales, argent provenant des membres émigrés, etc.).

On constate indéniablement une recrudescence des cérémonies plus ou moins prévues et programmées d'avance. Le gouvernement comme les tour-opérateurs essayent parfois de contrôler ou même de décider des dates et des heures des cérémonies importantes. L'Office du tourisme propose parfois aux familles de leur payer une certaine somme d'argent en échange, soit de fixer les conditions du déroulement de la cérémonie, soit d'avoir des informations jugées suffisantes concernant la fête. D'un point de vue pratique, certaines familles préfèrent réunir l'ensemble des membres et des invités pour deux ou trois cérémonies en même temps; elles font ainsi des économies en organisant moins de fêtes mais elles peuvent aussi en préparer de plus prestigieuses. Bref, les officines politiques, religieuses et touristiques travaillent de concert pour réinventer selon leurs intérêts respectifs les traditions locales... plus forcément très "traditionnelles »! Ici comme ailleurs dans l'archipel, l'indonésianisation poursuit son lissage des masses sur fond d'uniformisation culturelle menée au nom de l'unité nationale.

À l'occasion par exemple de la campagne officielle pour les élections en Indonésie (du 27 avril au 23 mai 1997), le mot d'ordre gouvernemental appliqué à Rantepao fut "pas de fêtes funéraires ou autres durant cette période». Les touristes en eurent pour leurs frais, mais cette attitude montre aussi que le politique (ou l'idéologique) importe en Indonésie encore davantage que l'économique même lorsqu'il s'agit de la sacro-sainte industrie du tourisme. La construction ou plutôt la consolidation de l'État-nation indonésien est à ce prix... Officiellement donc, aucune cérémonie n'a eu lieu au cours de la campagne électorale à Tana Toraja, mais certaines fêtes parvinrent cependant à transgresser les directives politiques. Comment? En prétextant de l'urgence de la tenue de la cérémonie pour diverses raisons et en occupant une position politique suffisamment élevée afin de pouvoir faire entendre sa voix et ainsi contourner les voies officielles. Toujours est-il que la politique lä̈que et la tradition religieuse font rarement bon ménage!

Devant les succès enregistrés par la religion chrétienne en terre toraja, les mythes issus de la seule tradition orale se transmettent moins entre les générations et, souvent, ils se modernisent ou s'actualisent en fonction des aspirations du moment, en perdant généralement leur sens symbolique. Les fêtes funéraires deviennent également plus importantes, car elles sont davantage demandées, plus populaires, au détriment des Rambu Tuka'qui, à l'exception notable des Mangrara Banua ou fête d'inauguration/rénovation d'un tongkonan (maison d'origine toraja), disparaissent lentement de la mémoire collective du peuple toraja. Ainsi, avons-nous pu constater que 
de nombreux jeunes gens de la région n'arrivent pas ou plus à distinguer correctement les différents rites les uns des autres, et la signification profonde des rituels, qu'elle soit d'ordre spirituel ou symbolique, semble désormais leur échapper. On assiste ces dernières années à une regrettable folklorisation des cérémonies traditionnelles, parfois ouvertement encouragée par des dirigeants politiques "plus indonésiens que toraja » et des leaders religieux locaux «plus chrétiens que aluk»...

L'anthropologue Kathleen Adams constate que «des funérailles réussies sont celles qui non seulement attirent beaucoup d'invités étrangers mais procurent nombre de documents écrits et audiovisuels. En résumé, les émigrés des centres urbains, les liens plus affermis entre les familles et les structures bureaucratiques du gouvernement et de l'Église, l'ouverture aux médias, et la présence des touristes et des anthropologues ont tous contribué à l'enrichissement et à la reformulation chez les Toraja de leurs traditions mortuaires. Aujourd'hui, les rites funéraires toraja sont un produit de ce dialogue avec le monde extérieur» (Adams, 1993, p. 70). Avec les cérémonies reformulées à la lumière de la modernité et de la mondialisation, la montée de toutes les formes de christianisme - y compris les plus intolérantes - dans la région, que reste-t-il de l'aluk todolo et de ses véritables chances de survie? Adams rend également compte de la perte d'influence du sentiment religieux - nous dirions plutôt de spiritualité - chez les Toraja, une perte dont le tourisme international porte une grande part de responsabilité :

[...] le discours influent des Toraja avec le monde n'a pas mis seulement l'accent sur la valeur distractive des funérailles, mais a engendré une dévaluation sur le plan spirituel parmi la population. Il semble que l'avis des touristes estimant que Tana Toraja n'est pas le Club Med mais le Club Dead n'est pas entièrement faux. Comme le Club Med, les modernes rites funéraires toraja impliquent une organisation parfaite et complexe en matière de divertissements et une préoccupation de toujours satisfaire les invités (Adams, 1993, p. 69).

Finalement. Tana Toraja, destination de choix pour fervents adeptes du tourisme religieux? Peut-être. Mais, alors que pour certains il est bien trop tôt pour l'affirmer, d'autres estiment qu'il est déjà trop tard. Pour le dire, et parfois même pour agir... Bien davantage que le spectre acculturateur du tourisme international, le processus actuel de christianisation, non seulement des fêtes mais également des mœurs toraja, ne porte plus simplement ombrage aux pratiques rituelles ancestrales, il risque d'accélérer la date d'échéance de la disparition programmée de l'aluk todolo dans l'agenda de l'Histoire... Surtout lorsqu'à ce processus s'accole une politique d'indonésianisation que rien ne semble pouvoir freiner.

Le problème crucial aujourd'hui consiste pour beaucoup de Toraja à enrayer la progression inquiétante des sectes protestantes d'obédience américaine, en particulier les pentecôtistes qui sont bien implantés et très actifs à Tator. À la recherche d'indépendance, les prédicateurs pentecôtistes multiplient les succès auprès de la population locale, en particulier chez les pauvres et les basses castes, cela pour plusieurs raisons: les mouvements religieux pentecôtistes sont résolument contre certains aspects des fêtes (sacrifices, consommation d'alcool et de différents aliments, tabac, combats de buffles et de coqs...) et profitent aussi d'une période d'essoufflement - liée à la sécularisation de l'institution et à l'enrichissement d'une partie de ses membres - au sein de la communauté protestante de tendance majoritaire (Gereja Toraja). Ils offrent la possibilité à une personne de bas statut de "s'élever socialement » de manière bien plus rapide ${ }^{12}$ qu'il ne lui serait possible de le faire si elle était protestante «classique» ou catholique, musulmane ou aluk; ils permettent dans la foulée de faire des économies conséquentes concernant les dépenses normalement occasionnées lors des cérémonies. L'argent économisé permet très souvent de remplir les caisses puis d'entreprendre la construction d'églises monumentales ou encore d'aider les plus démunis ${ }^{13}$. Planche de salut pour les Toraja déshérités, le pentecôtisme a, semble-t-il, de beaux jours devant lui en pays toraja, cela au détriment des rites et des fêtes qui risquent de tomber dans l'oubli ou plutôt de se modifier encore plus rapidement. Depuis une dizaine d'années, les touristes peuvent déjà «admirer » les versets bibliques inscrits sur des pancartes devant chaque chambre-tongkonan d'un hôtel à Rantepao...

En 1997, plusieurs événements accélérèrent en quelque sorte ce qu'il conviendrait de nommer la modernisation touristique du pays toraja. Une modernisation imposée « d'en haut» (Jakarta plutôt que les anciens dieux!) qui ne fait pas l'économie d'une appropriation de la religion des ancêtres à des fins purement commercialo-touristiques. Ainsi, le Bupati en poste, Tarsis Kodrat, à la suite du séminaire sur le développement du tourisme à Tator qui s'est tenu dans la région en octobre 1996, lance la première semaine culturelle toraja à partir de l'année 1997 (du 30 juin au 7 juillet) : «Le festival culturel se terminera par la grande cérémonie funéraire du Puang Sappang le 7 juillet $1997^{14}$.» La politique récupère officiellement un évènement religieux, le tout sur le dos de la culture! Ces prestigieuses funérailles princières se doivent d'être strictement ancrées dans les traditions et les cultes autochtones (le défunt était aluk à la date de sa mort, et verra son tau-tau trôner en compagnie des autres ancêtres divinisés dans la célèbre falaise mortuaire royale de Suaya). Pourra-t-elle se dérouler selon les préceptes précis et compliqués relevant entièrement de l'aluk todolo, alors qu'une foule de journalistes, d'ethnologues, de dirigeants politiques et économiques, de touristes étrangers et nationaux, se presseront aux portes du village et du rante (champ sacrificiel où se déroule la cérémonie) ? Les nombreux invités se pencheront sur la dépouille royale qui sera peut-être aussi un peu celle de la religion que d'aucuns nomment toujours «animiste», comme pour rendre sa disparition encore plus inéluctable et donc la pousser dans sa tombe déjà prête... Les lambeaux de l'aluk todolo, mis de la sorte au service du tourisme international et de la politique économique et culturelle, conduiront-ils à sacrifier le culte des ancêtres sur l'autel de la consommation et de la modernité ou, au contraire, sauront-ils stimuler, voire parachever sa résurrection? N'est-ce pas ici pour l'aluk todolo un moyen de revendiquer son droit à l'existence? Une solution provisoire pour «rester dans la course » avec les autres religions? En pays toraja, la compétition religieuse est rude et éprouvante, et pour garder ses chances il importe de ne pas être absent sur la scène politico-touristique locale..

Et si le tourisme international, ultime roue de secours au chevet de la tradition spirituelle menacée, sauvait finalement l'aluk todolo des vitrines de musée? Les chrétiens toraja, dans leur souhait flagrant de prospérer grâce aux recettes du tourisme feraient bien d'y songer, car les touristes ne se déplacent pas à Tana Toraja pour y rechercher le christianisme! Sans doute faudra-t-il choisir entre ces deux formes modernes de salut assuré que sont le ChristRoi et le Dieu-Dollar... Là aussi, le sacré et le marché ne peuvent plus rien sans l'aval ou, mieux, la complicité, du politique...

En 2006, inexorablement, les fondements spirituels et traditionnels de la société toraja s'effritent au risque de disparaître pour de bon au profit d'un moneytheism/monothéisme diffusé à grande échelle par des églises chrétiennes sans scrupules, avides de pouvoir séculier, autrement dit politique, dans une région en proie à de fortes mutations. L'alliance stratégique entre pouvoir politique et pouvoir clérical, tout en utilisant la culture comme outil et même comme arme identitaire, décide de l'avenir des habitants sans réellement les consulter, 
et sans leur laisser le moindre espoir de voir demain se préserver le sens originel des traditions locales.

\section{Bibliographie}

ADAMS, K.M. (1993). "Club Dead, Not Club Med: Staging Death in Contemporary Tana Toraja (Indonesia) », Southeast Asian Journal of Social Science, vol. 21, $\mathrm{n}^{\circ} 2$.

ALISJAHBANA, S.T. (1966). Indonesia: Social and Cultural Revolution, Kuala Lumpur, Oxford University Press.

BIGALKE, T.W. (1981). A Social History of Tana Toraja, 1870-1965, thèse, Madison, University of Wisconsin-Madison.

CRYSTAL, E. (1970). Toraja Town, thèse, Berkeley, University of California, Department of Anthropology.

DUVIGNAUD, J. (1977). Le don du rien. Essai d'anthropologie de la fête, Paris, Stock.

DUVIGNAUD, J. (1984). Fêtes et civilisations, Paris, Scarabée.

HUBERT, H. et M. MAUSS (1899). «Essai sur la nature et la fonction du sacrifice », L'Année sociologique, $\mathrm{n}^{\circ} 2$.

MENDE, T. (1954). L'Asie du Sud-Est entre deux mondes, Paris, Seuil.

MICHEL, F. (2000). Les Toraja d'Indonésie. Aperçu général socio-historique, Paris, L'Harmattan.

MICHEL, F. (1997). Tourisme, culture et modernité en pays toraja (Sulawesi-Sud, Indonésie), Paris, L'Harmattan, Coll. «Tourismes et Sociétés ».

VOLKMAN T.A. (1987). «Mortuary Tourism in Tana Tojara", dans R. S. Rodgers (dir.), Indonesian Religions in Transition, Tucson, University of Arizona Press.

VOLKMAN T.A. (1985). Feasts of Honor. Ritual and Change in the Toraja Highlands, Urbana, Uni. of Illinois Press.

WATERSON, R. (1993). «Taking the Place of Sorrow: The Dynamics of Mortuary Rites among the Sa'dan Toraja », Southeast Asian Journal of Social Science, vol. 21, $\mathrm{n}^{\circ} 2$.

\section{Notes}

1. Pour en apprendre davantage à ce sujet, lire F. Michel (1997), 285 p.

2. Sur l'histoire du peuple toraja jusqu'en 1950, voir F. Michel (2000), notamment p. 35-82.

3. Cité dans T. Mende (1954) p. 156-157. Kahar Muzdakkar sera en relation avec la rébellion conduite par le Darul Islam dirigé par Kartosuwiryo à Java-Ouest. À Sulawesi, des actes de guérilla se perpétuent en fait jusqu'en 1965, date de la capture et de la mort de Kahar Muzdakkar.

4. Rongne est sans doute parvenu à ce poste, car il était stratégiquement le mieux "placé» pour négocier avec Andi Sose; mais après d'incessantes difficultés, il en démissionnera en mars 1952.

5. Pour des discussions plus détaillées de ces aspects, voir T.W. Bigalke (1981), p. 397-414

6.Ibid., p. 426. Sur cette élection, ses prémices et ses conséquences, voir. T.W. Bigalke (1981) p. 425-432. L'historien conclut cette section de sa thèse en disant: «L'ère du Parkindo est arrivée», p. 432.

7.Le Monde, «Du nouveau dans le jamais vu. Sacrifice-spectacle en Indonésie», le 5 janvier 1980, p. 13.

8. Sur la fête, lire notamment les ouvrages de J. Duvignaud (1977) et (1984). Pour les cérémonies funéraires toraja, consulter R. Waterson (1993), p. 73-96.

9. Sur le sacrifice, se référer à l'étude classique de H. Hubert et M. Mauss (1899) p. 29-128.

10. Voir. T.A. Volkman (1987), p. 161-167.

11. Voir ibid., p. 166. Nos propres observations confirment amplement celles de Volkman.
12. Par exemple en devenant pasteur pentecôtiste, poste honorable et accessible à tous sans distinction de classes ou de caste, situation à laquelle on peut parfois accéder en seulement quelques mois (après avoir «appris » et lu intensivement la Bible). Devenir pasteur de l'Église protestante officielle nécessite un minimum de deux ans d'études et souvent plus. Enfin, pour devenir To'minaa, c'est-àdire officiant de la religion autochtone (Aluk to dolo), les années d'apprentissage (il faut compter la difficulté de maîtriser un savoir diffus et encyclopédique et celle d'accéder aux arcanes des nombreuses pratiques rituelles, sans oublier l'apprentissage d'une langue rituelle à la fois secrète et ésotérique, etc.) se comptent plutôt par dizaines. Comment peut-on aujourd'hui encore vouloir devenir To'minaa quand, ailleurs, en deux mois seulement, on se voit offrir le statut envié de pasteur pentecôtiste et qu'au lieu de puiser dans le passé et la nature toraja, il suffit de se référer à un livre proposant des recettes «clés (du paradis) en mains » pour se voir assurer son propre salut et même celui des autres?

13. Une immense église pentecôtiste (Gereja Betel) se trouve depuis le début de l'année 1995 sur la route principale au centre de Rantepao; à l'origine, l'édifice à construire devait être une mosquée...

14. Entretien avec le Bupati Tarsis Kodrat, 7 avril 1997, Makale. Ce Bupati (sorte de préfet) est un militaire de l'ethnie bugis, majoritairement musulmane et ennemie héréditaire des Toraja. Mais lui, il est catholique (et non pas protestant, comme la majorité du peuple toraja de nos jours), ce qui en fait un parfait représentant de l'État indonésien, à la fois extérieur (Bugis) et intérieur (chrétien), local et global... 